\title{
Introduction à l'étude de la diffusion de la lumière par les rugosités des surfaces optiques
}

\author{
C. Amra
}

Laboratoire d'Optique des Surfaces et des Couches Minces, U.R.A. 1120, Ecole Nationale Superieure de Physique de Marseille, Av. Escadrille Normandie-Niemen, Domaine Universitaire de St Jerome, F-13397 Marseille cedex 13, France

\section{I-Introduction}

On s'intéresse à l'étude de la diffusion de la lumière par des surfaces planes ayant reçu un poli pour des applications optiques. Il s'agit donc d'une diffusion sans changement de longueur d'onde, simplement liée aux caractéristiques géométriques de l'échantillon éclairé. C'est par exemple ce que perçoit l'oeil d'un observateur qui assiste à la réflexion d'un faisceau laser sur un dioptre plan (fig.1): si l'expérience se déroule en salle "propre", il n'y a pratiquement pas de particules en suspension dans l'air et on ne voit pas la "trace" des faisceaux spéculaires (incident, réfléchi ou transmis); par contre, on perçoit très bien l'impact du faisceau au niveau de la surface éclairée, ceci parce qu'une partie de l'énergie est perdue en dehors des directions spéculaires et est collectée par l'oeil. Cette diffusion est due à la présence d'irrégularités de surfaces (rugosités) sur l'échantillon; le cas échéant, elle peut provenir d'inhomogénéités de structure dans le volume de l'échantillon.

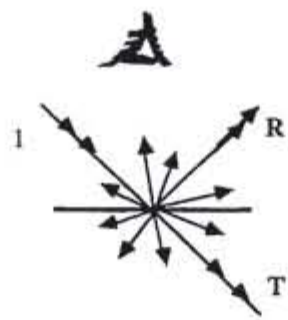

Fig.1: Diffusion par la rugosité d'un dioptre. (1), (R) et $(T)$ sont relatifs aux faisceaux spéculaires.

1- Généralités - définitions

En milieu homogène, la lumière se propage en ligne droite (principe de Fermat). Si le milieu est à gradient d'indice, il y a déviation continue du faisceau lumineux et l'énergie se trouve concentrée selon une courbe gauche dans l'espace. Par contre, en milieu inhomogène ou aléatoire, il y a perte d'énergie dans toutes les directions: c'est la diffusion de volume.

On se limite dans ce cours aux pertes par diffusion à la surface de séparation de deux milieux homogènes. L'échantillon est supposé macroscopiquement plan et est éclairé par un faisceau laser gaussien sous l'incidence moyenne $\mathrm{i}_{\mathrm{i}}$ (fig.2).
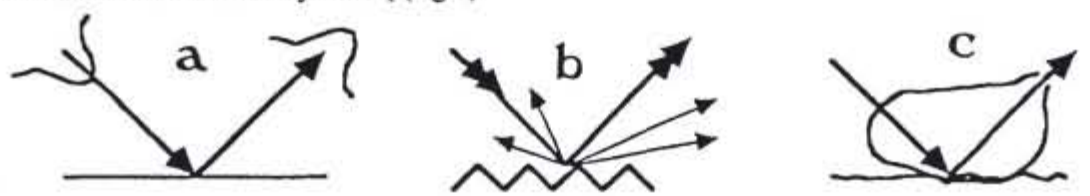

Fig.2: Réflexion spéculaire par un dioptre plan idéal (a), diffraction par un profil périodique (b) et diffusion par une surface aléatoire (c). 
Si le dioptre est idéalement plan (sans rugosités), le faisceau réfléchi est une gaussienne pondérée par le spectre du coefficient de réflexion de l'échantillon (fig.2-a). La direction moyenne $\mathrm{i}_{\mathrm{r}}$ de ce faisceau est donnée par les lois de Descartes $\left(\sin \mathrm{i}_{\mathrm{i}}=\sin \mathrm{i}_{\mathrm{r}}\right.$ ). Par contre, si le dioptre est une surface périodique (dite réseau) de pas c plus grand que la longueur d'onde $\lambda$ d'éclairement, on assiste à une discrétisation du faisceau réfléchi (fig.2-b): l'énergie lumineuse est concentrée selon quelques directions particulières appelées ordres de diffraction. Ces directions sont données par la loi des réseaux $\left(\sin \theta_{\mathrm{k}}=\mathrm{k} \lambda / \mathrm{c} \mathrm{a} \mathrm{i}=0^{\circ}\right)$. Enfin, si le dioptre présente des fluctuations de hauteur aléatoire (surface dite rugueuse), l'énergie est réémise selon une répartition spatiale continue dans tout l'espace, appelée indicatrice de diffusion (fig.2-c).

On remarquera que cette diffusion de surface s'apparente ainsi à la diffraction par un profil aléatoire. En effet, pour un réseau, le profil périodique se décompose en une suite discrète de sinusoïdes (série de Fourier) qui conduisent chacune à un ordre de diffraction particulier. Pour une surface aléatoire, le profil se décompose en une suite continue de sinusoïdes (intégrale de Fourier); il y a une infinité d'ordres de diffraction qui constituent alors l'indicatrice de diffusion.

2- Ordres de grandeur

Les rugosités (hauteurs quadratiques moyennes) des surfaces optiques n'excèdent pratiquement jamais 2 ou $3 \mathrm{~nm}$. Pour les meilleurs micro-polis que l'on peut obtenir sur verre, la rugosité est de l'ordre de $0,2 \mathrm{~nm}$ et conduit à des pertes totales par diffusion de quelques $10^{-6} \mathrm{du}$ flux incident (ceci pour des surfaces parfaitement propres). En fait, dans la plupart des systèmes optiques, chaque composant de base (lentille, miroir, prisme, filtre, ...) est aujourd'hui recouvert d'un traitement multicouche permettant d'accroître ou de réduire le facteur de réflexion ou de transmission de la lumière. Ces empilements de couches minces viennent modifier l'amplitude et la répartition spatiale des flux diffusés. Les indicatrices de diffusion dépendent alors de phénomènes interférentiels entre les différentes sources de diffusion aux interfaces rugueuses.

Le dépôt d'une couche unique peut conduire à une augmentation ou à une diminution de la diffusion. Les pertes sont en général de l'ordre de $10^{-4}$ pour les anti-reflets. Pour les miroirs multidiélectriques, ces pertes sont presque toujours supérieures à $10^{-4}$. Enfin, pour les filtres interférentiels à bande étroite (du type Fabry-Perot), les pertes minimales sont de l'ordre de $10^{-3}$. Ces ordres de grandeur sont donnés pour des conditions optimales de réalisation d'empilements multicouches. Dans le cas général, ces valeurs dépendent des matériaux utilisés et de la technique de dépôt associée. Chaque matériau est en effet susceptible de reproduire les défauts du substrat et d'ajouter sa propre rugosité intrinsèque.

\section{3-Intérêt - applications}

Les applications liées à l'étude de la diffusion sont nombreuses, et interviennent en général lorsque l'on veut établir un bilan énergétique détaillé (typiquement à mieux que $10^{-3}$ ). Il faut écrire en effet : $1=R+T+A+D$, où $R$ et $T$ sont les facteurs de réflexion et de transmission du dioptre ou de l'empilement, $A$ les pertes par absorption intrinsèque, liées à la partie imaginaire de l'indice de réfraction des matériaux, et $D$ les pertes par diffusion. Dans certains cas, la diffusion est supérieure à l'absorption et vient fausser la détermination des coefficients d'extinction des matériaux; en effet, une mesure de pertes par photométrie donne accès à la grandeur $1-\mathrm{R}-\mathrm{T}=\mathrm{A}+\mathrm{D}=\mathrm{A}^{\prime}$ qui est l'absorption apparente, et qui peut n'être que la conséquence de rugosités de surface (cas où $\mathrm{A} \ll<\mathrm{D}$ ).

De nombreux systèmes optiques requièrent des composants avec des pertes minimales par diffusion. C'est par exemple le cas des miroirs pour gyrolaser, où l'on cherche à obtenir un facteur de réflexion superrieur à $1-10^{-4}$ (il faut donc $A^{\prime}=A+D<10^{-4}$ ). De même, pour les anti-reflets, on demande parfois une réflexion inférieure à $10^{-4}$. La diffusion peut également contribuer à augmenter l'absorption et 
favoriser ainsi le claquage des traitements optiques soumis à des flux intenses (problèmes d'endommagement laser). Enfin, pour les systèmes multi-démultiplexeurs couramment utilisés en télécommunications optiques, il y a augmentation de la diaphonie à cause des pertes par diffusion. La plupart de ces systèmes utilisent des filtres interférentiels à bande étroite qui sont constitués de filtres Fabry-Perot mis en série. Pour obtenir une bande spectrale étroite et des taux de réjection élevés, le filtre doit être très résonnant (champ électrique intense dans le volume du composant) et ceci conduit à des pertes intenses par diffusion (fig.3).

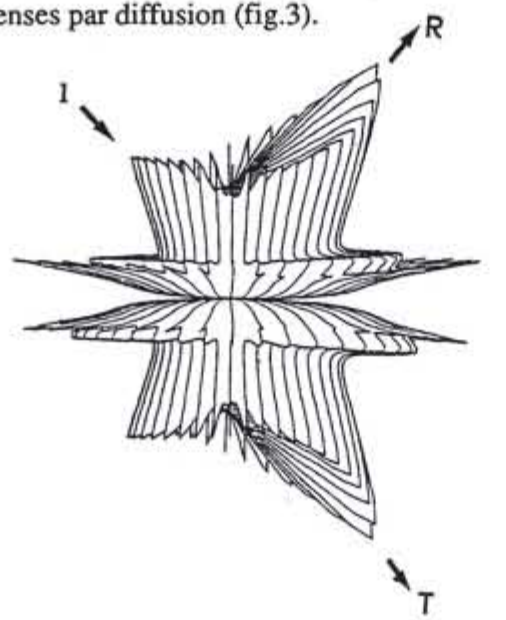

Fig.3: Indicatrice de diffusion par un filtre interférentiel d bande étroite. $R$ et $T$ sont les facteurs de réflexion et de transmission du filtre. On remarquera la présence d"'anneaux de diffusion".

En figure 4, on trouve un exemple de démultiplexeur utilisant de tels filtres; un calcul classique ne faisant intervenir que les directions spéculaires conduit à une isolation de 10-10 entre les deux voies de télécommunications $\lambda_{1}$ et $\lambda_{2}$. Par contre, la mesure donne une isolation de quelques $10^{-6}$, ceci parce que la lumière diffusée passe directement d'un canal de transmission à l'autre.

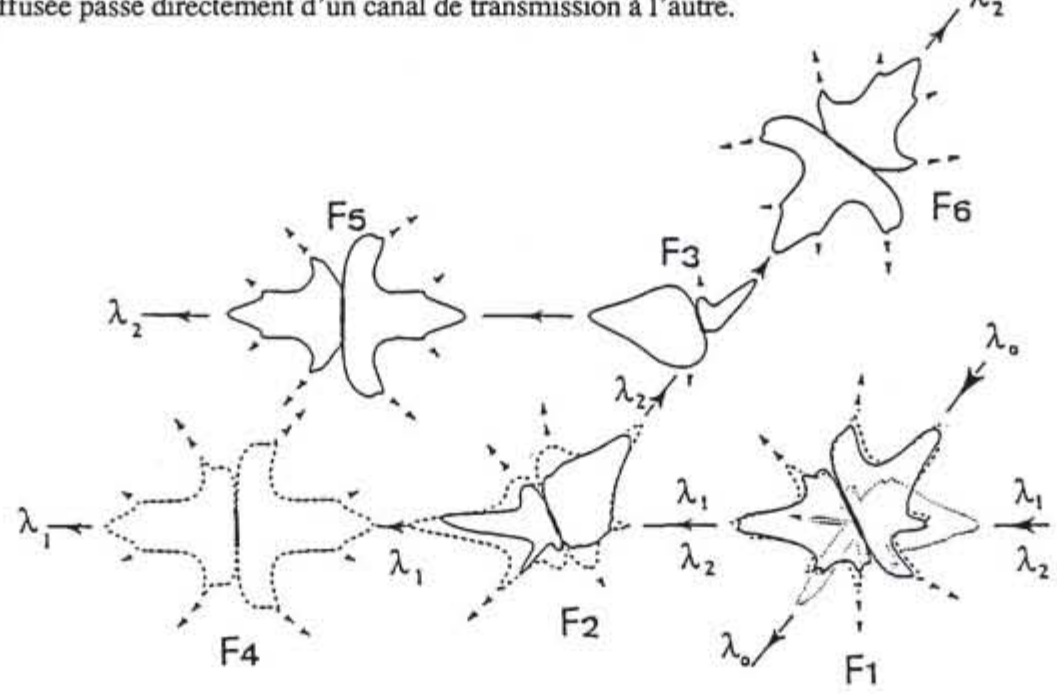

Fig.4: Séparateur spectral utilisant deux voies de télécommunication $\lambda_{1}$ et $\lambda_{2}$. Six filtres interférentiels ( $\left.F_{i}\right)$ permettent de séparer 3 longueurs d'onde $\lambda_{i}$. Pour chaque filtre et chaque longueur d'onde, on a représenté l'indicatrice de diffusion en trait plein $\left(\lambda_{2}\right)$, traits espacés $\left(\lambda_{l}\right)$ et en pointilles $\left(\lambda_{0}\right)$. 
D'une façon plus générale, la diffusion étant due à des hétérogénéités de structure au niveau des interfaces ou du volume des composants, elle transporte une information relative à la microstructure (à l'échelle des fréquences spatiales optiques) de ces composants. Cette information peut s'extraire si l'on dispose d'un modèle théorique adéquat et mettant en jeu un nombre restreint de paramètres (résolution du problème inverse).

\section{II-Techniques de mesures}

Les polisseurs ont besoin d'outils pour caractériser les micropolis. On distingue essentiellement les mesures dans l'espace direct (repère classique Oxyz) qui donnent immédiatement accès au profil de rugosité, et les mesures dans l'espace de Fourier (mesures de diffusion optique) qui donnent accès au spectre (ou transformée de Fourier) des défauts du micro-poli.

\section{1-Mesures dans l'espace direct}

Il s'agit en général d'un stylet mécanique qui se déplace sur l'échantillon en épousant son profil. A partir de l'échantillonnage obtenu, on calcule les différentes grandeurs (fonction d'autocorrélation, spectre de rugosité,...) permettant de décrire la statistique de l'état de surface. Quelques appareils haut de gamme (du type Talystep) utilisent un stylet de rayon de courbure $0,2 \mu \mathrm{m}$, ce qui garantit une bonne résolution latérale. Pour d'autres appareils, le stylet mécanique est remplacé par un faisceau laser fortement focalisé (spot de l'ordre du micron), les mesures s'obtenant à l'aide d'une détection hétérodyne.

Ces techniques de profilométrie présentent toutefois deux inconvénients: d'une part, la mesure s'effectue selon une direction particulière sur l'échantillon, ce qui ne permet pas de détecter une éventuelle anisotropie de la rugosité (orientation particulière des défauts); d'autre part, on n'a pas accès aux pertes par diffusion, lesquelles doivent alors être reconstruites à partir d'un modèle théorique adéquat.

\section{2-Mesures dans l'espace de Fourier}

Il s'agit de mesurer la répartition spatiale de l'énergie diffusée dans tout l'espace, en dehors des directions spéculaires. Ces appareils sont appelés "diffusomètres" et un exemple en est donné en figure 5.

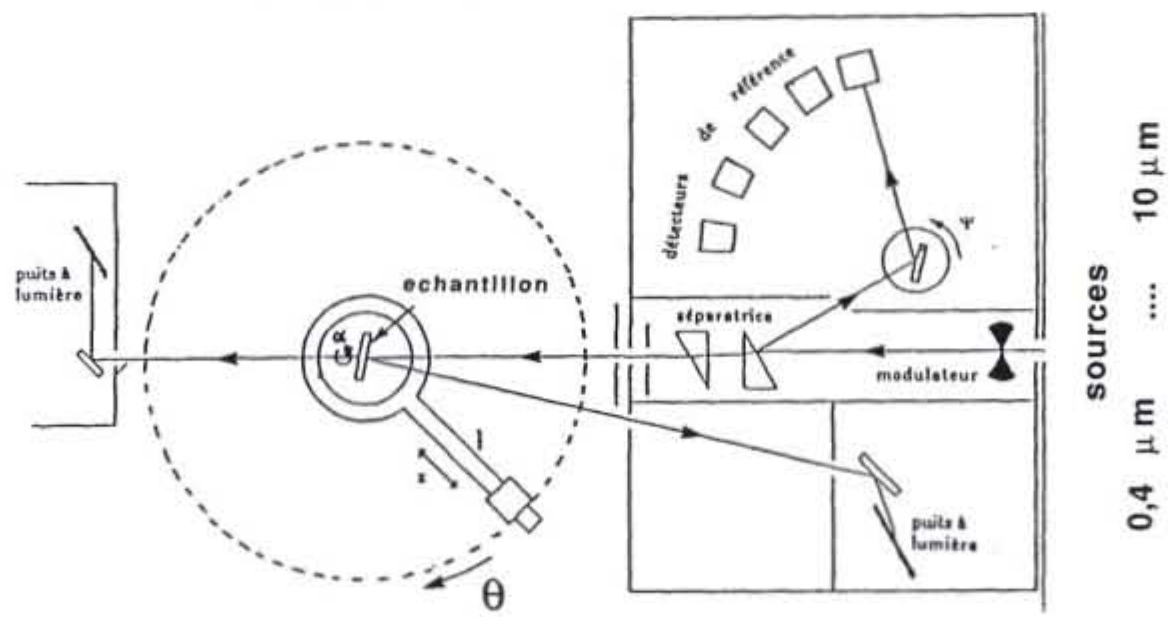

Figs: Schéma de base du dispositif de mesure de la diffusion. 
Le principe de la mesure est le suivant: l'échantillon fixé dans un plan vertical est éclairé en incidence quasi-normale $\left(\mathrm{i}=1,5^{\circ}\right)$ par un laser $\mathrm{He}-\mathrm{Ne}$ (puissance $4 \mathrm{~mW}$, longueur d'onde $633 \mathrm{~nm}$ ). Le diamètre de la surface éclairée est d'environ $2 \mathrm{~mm}$. Les faisceaux réfléchis et transmis sont éliminés à l'aide de puits a lumiere constitués de verres opaques superpolis sous incidence de Brewster. Un bras mobile de longueur $1 \mathrm{~m}$ supporte une fibre optique et permet d'effectuer des mesures de diffusion angulaire $\mathrm{I}(\boldsymbol{\theta})$ dans le plan d'incidence horizontal. L'échantillon est de plus animé d'un mouvement de rotation $\alpha$ autour de sa normale, ce qui permet d'enregistrer une indicatrice $\mathrm{I}(\theta, \alpha)$. Cette indicatrice correspond aux pertes réelles dans tout l'espace; il est en effet équivalent, en incidence normale et en lumière naturelle, de faire tourner l'échantillon autour de sa normale ou de déplacer le détecteur sur un cône centré sur cette normale. L'angle $\alpha$ est donc un angle polaire caractérisant la position d'un plan particulier de diffusion ( $\alpha=0$ et $\pi / 2$ sont les plans horizontaux et verticaux pour le dispositif expérimental).

En figure 6, on donne un exemple d'indicatrice mesurée avec ce système, pour un échantillonnage de $3^{\circ}$ en $\theta$ et de $3,6^{\circ}$ en $\alpha$. Les 3000 points de mesure sont obtenus en $20 \mathrm{mn}$.

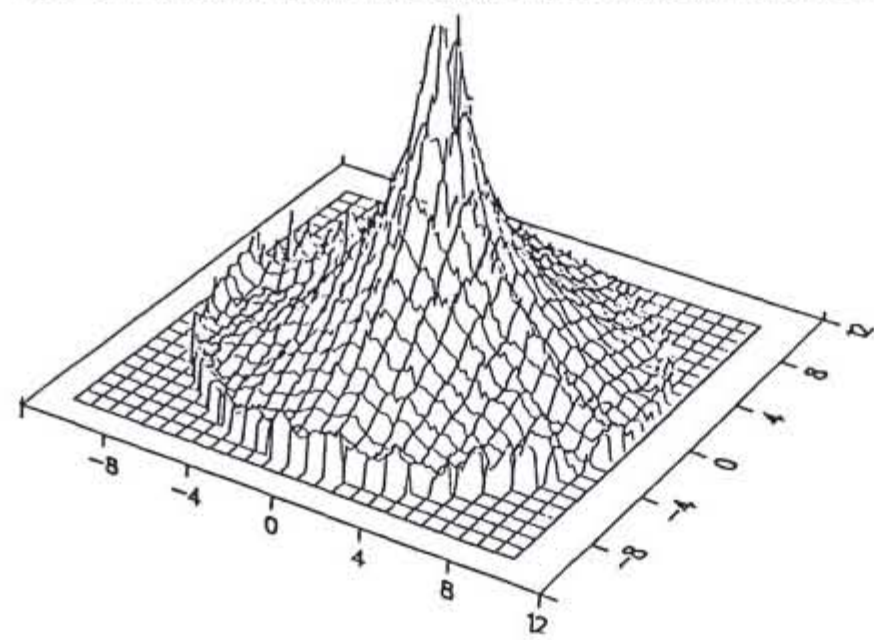

Fig.6: Exemple de mesure d'une indicatrice de diffusion en réflexion, pour une incidence d'éclairement normale. Le plan "quadrillé" est parallele au plan moyen de la surface rugueuse. Les faisceaux spéculaires ne sont pas pris en compte. Les axes sont gradućs en fréquences spatiales $\left(\sigma_{x}=\sigma \cos \alpha\right.$, $\sigma_{y}=\sigma \sin \alpha$ old $\left.\sigma=(2 \pi / \lambda) \sin \theta\right)$, avec pour unité le $\mu \mathrm{m}^{-1}$.

En général on se contente d'une section moyenne sur l'angle polaire $\alpha$. C'est le cas de la figure 7 relative à l'influence du nettoyage.

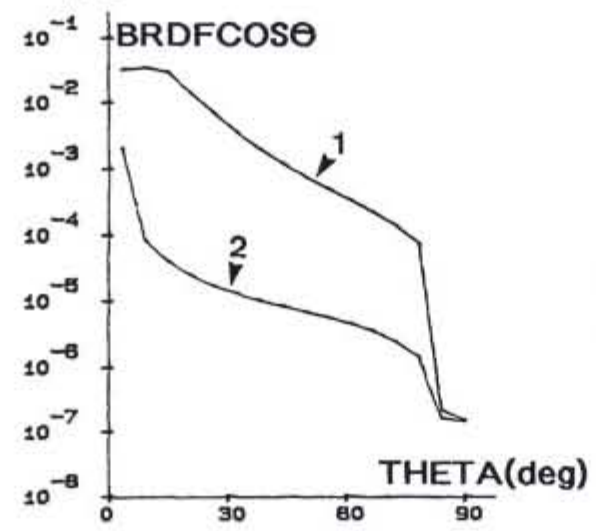

Fig.7: Section plane moyenne de lindicatrice de diffusion avant (1) et apress (2) nettoyage de l'échantillon. 
La courbe (1) est la mesure d'un échantillon à la sortie de l'atelier de polissage. La diffusion totale est élevée $\left(1,8.10^{-2}\right)$ et provient de résidus de produits de polissage. La courbe (2) est obtenue après nettoyage du même échantillon; les pertes sont plus faibles $\left(9.10^{-5}\right)$ et sont dues à la rugosité de l'état de surface.

\section{Angle minimum de mesure}

Il est important de savoir à partir de quel angle le signal mesuré est effectivement caractéristique des défauts du micropoli, et non pas de la répartition spatiale du faisceau spéculairement réfléchi. Pour cela il faut reconstituer le faisceau réfléchi à partir de la mesure de l'intensité angulaire du faisceau incident. On procède de la façon suivante: tout faisceau réel se décomposant en une somme dite paquet d'ondes planes, le champ électrique s'écrit en lumière monochromatique:

$$
\left.\vec{E}^{+}(\vec{\rho})=\int_{\vec{\beta}} \vec{A}+(\vec{\beta}) e^{\vec{k}+(\vec{\beta}) \cdot \vec{\rho}} d \vec{\beta} \text { où } \vec{\rho}=\overrightarrow{(r}, z\right) \text {, et } \vec{A}^{+}(\vec{\beta}) \text { est l'amplitude de l'onde plane associée à }
$$

la fréquence spatiale $\vec{\beta} / 2 \pi$ caractéristique de la direction $(i, \psi): \vec{\beta}=k \sin i \mid \begin{aligned} & \cos \psi \\ & \sin \psi\end{aligned}$ avec $k=\frac{2 \pi}{\lambda}$.

Le vecteur d'onde correspondant s'écrit: $\vec{k}^{+}(\vec{\beta})=\mid \begin{aligned} & \vec{\beta} \\ & \alpha(\beta)=\sqrt{k^{2}-\beta^{2}}\end{aligned}$.

Le flux total transporté à travers un plan perpendiculaire à la direction moyenne du faisceau s'écrit, à une constante de proportionnalité près: $\phi^{+}=\int_{\beta^{2}} \alpha(\beta)\left|\vec{A}^{+2}(\vec{\beta})\right| \mathrm{d} \vec{\beta}$, et l'intensité angulaire incidente est donnée par: $\mathrm{I}^{+}(\mathrm{i}, \psi)=\mathrm{d} \phi^{+} / \mathrm{d} \Omega=\mathrm{k}^{3} \mathrm{~A}^{2}(\mathrm{i}, \psi) \cos ^{2} \mathrm{i}$ où $\mathrm{d} \Omega=\sin \mathrm{i}$ di $\mathrm{d} \Psi$ est un angle solide élémentaire. Si l'on sait donc mesurer cette répartition $\mathrm{I}^{+}$, le flux réfléchi par un dioptre supposé idéalement plan s'obtient grâce à une pondération par le spectre $R(\vec{\beta})$ du coefficient de réflexion du dioptre:

$$
\phi^{-}=\int_{\vec{\beta}^{2}} \alpha(\beta) R(\vec{\beta})\left|\vec{A}^{+}(\vec{\beta})\right|^{2} \mathrm{~d} \vec{\beta} \Leftrightarrow I^{-}(i, \psi)=R(i) I^{+}(i, \psi)
$$

Dans ces conditions, le signal mesuré n'est caractéristique de la rugosité que dans le domaine angulaire où la diffusion est supérieure à cette répartition spéculaire $\mathrm{I}^{-}$(fig.8).

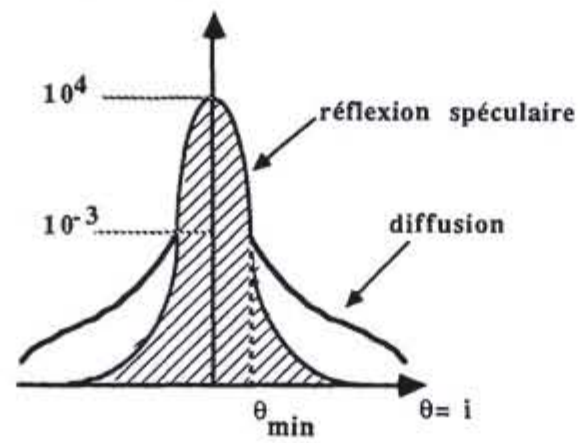

Fig.8: Réflexion spéculaire (zone hachurée) et diffusion. L'angle $\theta_{\text {min }}$ est l'angle minimum de mesure de la diffusion.

D'un point de vue pratique, le faisceau est peu divergent $(\Delta \mathrm{i}=1 \mathrm{mrad})$ et $\mathrm{R}(\mathrm{i})$ est constant sur le domaine utile $\Delta \mathrm{i}$. La mesure de $\mathrm{I}^{+}(\mathrm{i}=\theta$ ) s'effectue au diffusomètre en l'absence d'échantillon (pas de faisceau réfléchi): c'est la courbe (1) de la figure 9. Près du faisceau incident $\left(\theta=0^{\circ}\right)$, le détecteur reçoit une diffusion due à l'impact du faisceau sur le puits à lumière en transmission. En dehors des voisinages $\theta=0^{\circ}$ et $\theta \approx 180^{\circ}$, le signal est caractéristique du bruit de fond du récepteur. Près du faisceau transmis $\left(\theta=180^{\circ}\right)$, l'accroissement du signal est lié au fait que l'on mesure les "pieds" de la gaussienne du faisceau laser; en 
fait on mesure également la diffraction par tous les diaphragmes ainsi que la diffusion par les particules en suspension dans l'air (bien que le système de mesure soit en salle propre, il subsiste quelques particules responsables d'une diffraction au voisinage immédiat du faisceau transmis).

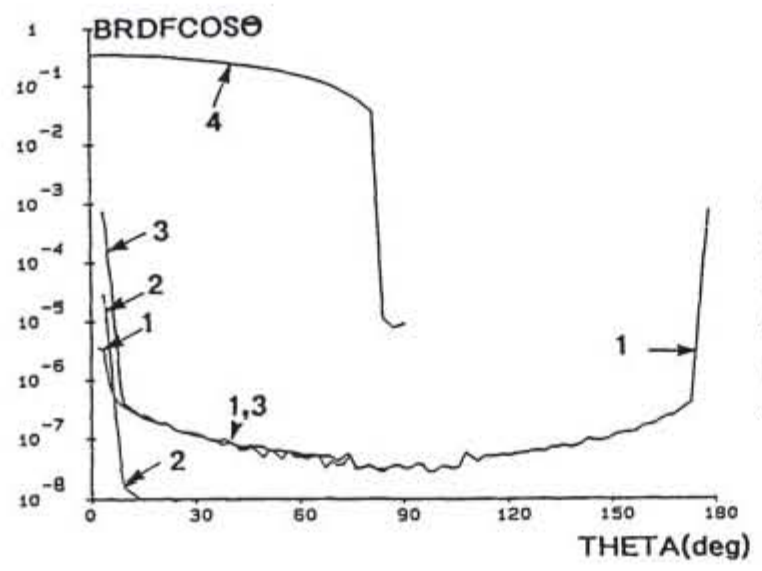

Fig.9: Réponse d vide du diffusometre. (1) est la courbe mesurée en l'absence d'échantillon. (2) et (3) sont les réflexions spéculaires reconstituées pour des échantillons de réflexion $R=0,04$ (courbe 2) et $R=0,99$ (courbe 3). (4) est la diffusion d'un échantillon lambertien.

A partir de cette réponse à vide (courbe 1), on reconstitue la lumière parasite en présence d'un échantillon de réflexion $\mathrm{R}=0,04$ (courbe 2 ) ou $\mathrm{R}=0,99$ (courbe 3 ). La courbe (4) est relative à un échantillon lambertien de réflexion diffuse proche de l'unité. La figure 9 permet ainsi de mettre en évidence la détectivité et la dynamique du système dans tout le domaine angulaire de mesure.

\section{IV-Techniques de calcul}

Pour caractériser l'état de surface à partir des mesures de diffusion, il faut disposer d'un modèle théorique adéquat. On distingue essentiellement les modèles scalaires, en général basés sur les lois de l'optique géométrique, et les modèles vectoriels, issus de l'électromagnétisme. Nous avons vu que les rugosités $\delta$ des micropolis n'excèdent pas $3 \mathrm{~nm}$ et sont donc faibles devant les longueurs d'onde $\lambda$ visibles; par conséquent, un modèle au $1^{\mathrm{er}}$ ordre suffit largement pour notre application $(\delta / \lambda<1)$. L'avantage de ce type de modèle est qu'il permet de résoudre le problème inverse, c'est-à-dire l'extraction, à partir de la mesure, des paramètres caractéristiques de l'état de surface.

\section{1-Modèle scalaire}

La formule suivante a été largement utilisée : $\mathrm{D}=\mathrm{R}(4 \pi \delta / \lambda)^{2}$, où $\mathrm{D}$ représente les pertes totales par diffusion (intégrées dans tout l'espace), et $\mathrm{R}$ le facteur de réflexion du dioptre en l'absence de rugosité. $\delta$ et $\lambda$ désignent respectivement la rugosité de l'échantillon et la longueur d'onde d'éclairement. Le rapport D/R est également connu sous le nom de T.I.S. (Total Integrated Scattering). On voit donc qu'une simple mesure de diffusion totale (avec des appareillages du type sphère intégrante), jointe au calcul du coefficient de réflexion idéal, donne accès à la rugosité. Toutefois, cette rugosité est une grandeur intégrée qui donne peu d'informations sur l'état de surface (longueur d'autocorrélation, anisotropie, pseudo-périodicités ..?). De plus, il est prudent de l'écrire sous la forme $\delta(\lambda)$ pour rappeler qu'elle dépend de la longueur d'onde: en effet, les classes de défauts responsables de la diffusion à $10,6 \mu \mathrm{m}$ n'ont rien à voir avec celles responsables de la diffusion dans le domaine visible. Précisons enfin que cette formule scalaire est établie 
en supposant la longueur d'autocorrélation $L$ des défauts grande devant la longueur d'onde $(L / \lambda \gg 1)$, c'est-à-dire pour une diffusion concentrée aux très faibles angles. La notion de T.I.S. a cependant rendu de nombreux services et montre par exemple que la diffusion croît avec le facteur de réflexion (ceci pour un dioptre et en première approximation).

\section{2-Modèle vectoriel}

On sait ramener le problème du dioptre rugueux à celui d'un dioptre idéalement plan mais recouvert de courants électrique et magnétique de surface. La résolution des équations de Maxwell en présence de ces sources conduit au résultat suivant:

$\mathrm{I}(\theta, \phi)=\mathrm{C}(\theta, \phi) \gamma(\theta, \phi)$ où $\mathrm{I}(\theta, \phi)$ est le flux diffusé par unité d'angle solide, normé au flux incident. Le couple $(\theta, \phi)$ caractérise une direction de diffusion (fig.10).

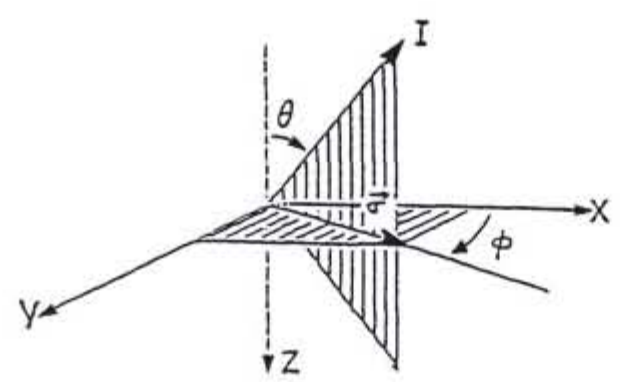

Fig.10: Définition des angles $(\theta, \phi)$ caractérisant une direction de diffusion. $z=0$ est le plan moyen de la surface rugueuse.

Si l'on considère la surface diffusante comme une source secondaire, I représente l'intensité photométrique. Il est d'usage également d'introduire la notion de BRDF (Bidirectional Reflectance Distribution Function): $\quad \mathrm{I}(\theta, \phi)=\mathrm{BRDF}(\theta, \phi) \cdot \cos \theta$, où $\mathrm{BRDF}$ joue le rôle d'une luminance $\left(B R D F(\theta)=R^{d} / \pi\right.$ pour un échantillon lambertien de réflexion diffuse $\left.R^{d}\right)$.

Le coefficient $\mathrm{C}(\theta, \phi)$ est un terme optique qui ne dépend que des indices des milieux homogènes et des conditions d'illumination et d'observation (longueur d'onde, incidences, polarisations). Le terme $\gamma(\theta, \phi)$ est appelé spectre de rugosité et contient toute l'information accessible sur l'état de surface; c'est la transformée de Fourier de la fonction d'autocorrélation des défauts du micropoli. En termes de fréquences spatiales $\vec{\sigma}=k \sin \theta \mid \begin{aligned} & \cos \phi \\ & \sin \phi\end{aligned}$, on voit donc que la mesure de la diffusion angulaire, jointe au calcul du coefficient optique, donne accès au spectre de rugosité: $\quad \gamma(\vec{\sigma})=\frac{I(\vec{\sigma})}{C(\vec{\sigma})}$.

On donne, sous incidence normale et en lumière naturelle, pour la diffusion en réflexion:

$$
\mathrm{C}(\theta, \phi)=\mathrm{C}(\theta)=\frac{32 \pi^{4}}{\lambda^{4}} n_{0}^{4} \cos ^{2} \theta\left[\left|\mathrm{Q}_{\mathrm{s}}^{2}\right|+\left|\mathrm{Q}_{\mathrm{p}}^{2}\right|\right]
$$

avec:

$$
\mathrm{Q}_{\mathrm{S}}=\frac{(\sqrt{\varepsilon}-1)}{\left(\cos \theta+\sqrt{\varepsilon-\sin ^{2} \theta}\right)} \text { et } \mathrm{Q}_{\mathrm{p}}=\frac{(\sqrt{\varepsilon}-1) \sqrt{\varepsilon-\sin ^{2} \theta}}{\left(\varepsilon \cos \theta+\sqrt{\varepsilon-\sin ^{2} \theta}\right)}
$$

où $\varepsilon=\left(\mathrm{n}_{\mathrm{S}} / \mathrm{n}_{0}\right)^{2}, \mathrm{n}_{0}$ et $\mathrm{n}_{\mathrm{S}}$ désignant respectivement les indices optiques des milieux incident (en général l'air) et du substrat. 


\section{Application}

Pour vérifier que le spectre obtenu est une caractéristique intrinsèque au micropoli, il est intéressant d'effectuer des mesures pour différentes conditions d'éclairement. La mesure I et le calcul $\mathrm{C}$ sont alors différents, mais le rapport $\gamma=\mathrm{I} / \mathrm{C}$ doit rester constant (fig.11-a). Par contre, si l'échantillon est contaminé (problèmes de condensation sur le verre...), les rugosités ne sont pas la seule source de diffusion et les spectres se dissocient avec la polarisation de la lumière incidente (fig.11-b).
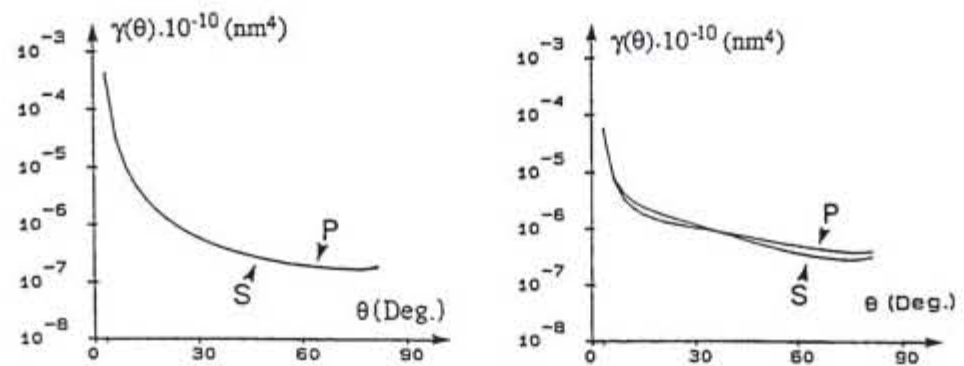

Fig.11: Mesure du spectre de rugosité pour deux polarisations (S et $P$ ) de la lumière incidente. La figure (b) est relative du un échantillon "contaminé".

Ceci constitue donc un test de propreté pour les échantillons. Ce test n'est cependant accessible que pour des échantillons opaques, pour lesquels on n'est pas gêné par la diffusion due aux faces arrière et latérales.

\section{La "fenêtre" optique}

Pour une diffusion angulaire mesurée à la longueur d'onde $\lambda$ dans le domaine angulaire $\theta_{\min }=0^{\circ} \leq \theta \leq \theta_{\max }=90^{\circ}$, le domaine de fréquences spatiales $v=\sigma / 2 \pi$ accessibles à l'expérience s'étale entre $v_{\min }=\sin \theta_{\min } / \lambda$ et $v_{\max }=1 / \lambda$. Avec le diffusomètre précédent, on a de façon courante: $\lambda=633 \mathrm{~nm}$, $\theta_{\min }=1,8^{\circ}, v_{\min }=0,05 \mu \mathrm{m}^{-1}$ et $v_{\max }=1,58 \mu \mathrm{m}^{-1}$. Le spectre de rugosité n'est donc connu que sur une fenêtre fréquentielle limitée et la rugosité s'obtient en intégrant le spectre sur ce domaine:

$$
\delta^{2}=\int_{\vec{\sigma}} \gamma(\vec{\sigma}) \mathrm{d} \vec{\sigma}=\mathrm{k}^{2} \int_{\theta, \phi} \gamma(\theta, \phi) \cos \theta \sin \theta \mathrm{d} \theta \mathrm{d} \phi
$$

Il faut donc se limiter à cette rugosité dite mesurable, qui caractérise les défauts responsables de la diffusion pour l'application considérée. La borne minimale $\sigma_{\min }$ est liée à l'angle minimum de mesure, et la borne maximale ne peut excéder $2 \pi / \lambda$ : au-delà de cette valeur, les fréquences spatiales correspondent à des réseaux sinusoïdaux de pas $c=2 \pi / \sigma$ inférieurs à la longueur d'onde et qui ne diffractent donc pas (il s'agit d'ondes évanescentes caractérisant une énergie confinée au voisinage immédiat de la surface); on dit pour cela que la lumière ne voit pas les défauts dont les dimensions latérales sont inférieures à la longueur d'onde.

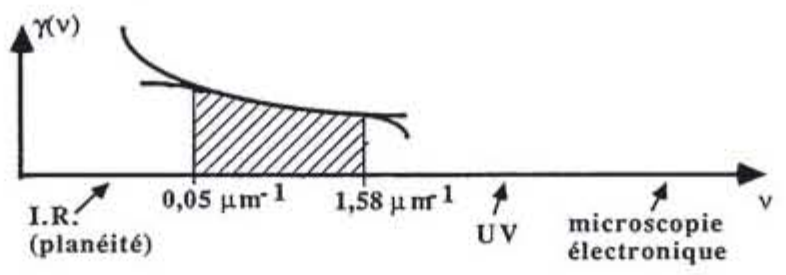

Fig.12: Fenètres fréquentielles. La zone hachurée est relative à la longueur d'onde $633 \mathrm{~nm}$. 
Il est clair que la bande fréquentielle et donc la rugosité varient avec la longueur d'onde (fig.12). Pour les mesures de diffusion dans l'infra-rouge lointain, la rugosité est relative à de faibles fréquences spatiales caractéristiques de la planéité, laquelle est responsable de la déformation du front d'onde dans le visible. Vers l'ultra-violet, on caractérise des défauts de plus courtes périodes spatiales. Remarquons enfin que la microscopie électronique donne accès à des fréquences spatiales très élevées mais qui ne permettent pas de prédire la diffusion optique; pour le même échantillon, on peut trouver une rugosité de $0,3 \mathrm{~nm}$ par loptique et de $10 \mathrm{~nm}$ par microscopie électronique.

\section{V-Comment voit-on la surface?}

On s'intéresse ici à la fonction de transfert de l'appareillage. Ceci est important si l'on veut pouvoir comparer les valeurs de rugosités obtenues par différentes techniques.

\section{1-Rappels- fonction d'autocorrélation}

Supposons que l'on veuille introduire une notion d'ordre relativement à un profil $\mathrm{h}(\mathrm{x})$ unidimensionnel et de support $\mathrm{T}$. Un observateur au point $\mathrm{M}(\mathrm{x})$ sur la surface aperçoit un certain paysage $h(x)$. Au point $M(x-\tau)$, il voit un autre paysage $h(x-\tau)$. Quelle ressemblance $y$ a-t-il entre ces deux paysages? Pour cela, on définit une distance $D(\tau)$ entre les paysages, au sens des moindres carrés:

$D(\tau)=\frac{1}{T} \int_{x}[h(x)-h(x-\tau)]^{2} d x$.Cette fonction D exprime une notion de non-ressemblance, puisqu'elle est nulle en $\tau=0$, c'est-à-dire lorsque les paysages sont identiques. Quand la variable $\tau$ croît, les paysages se différencient et la fonction $\mathrm{D}$ croit.

La fonction d'autocorrélation $\Gamma(\tau)=\frac{1}{T} \int_{x} h(x) h(x-\tau) d x$ s'écrit aussi: $\Gamma(\tau)=\Gamma(0)-\frac{1}{2} D(\tau)$ et exprime donc une notion de ressemblance ( $\Gamma$ est maximale à l'origine). Pour une surface purement aléatoire, $\Gamma$ est strictement décroissante en moyenne (fig.13-a) puisque la ressemblance diminue avec $\tau$. II est d'usage d'introduire la longueur d'autocorrélation $L$ définie par: $\Gamma(L)=1 / e \Gamma(0)$. Cela signifie qu'au delà d'une distance $\tau=\mathrm{L}$, les deux paysages n'ont pratiquement plus rien à voir entre eux (pour un bruit blanc idéal, $\mathrm{L}=0$ ). La présence d'éventuels maxima de $\Gamma$ est caractéristique de pseudo-périodicités sur l'échantillon, c'est-à-dire que l'on retrouve partiellement le paysage initial à une distance L' (fig.13-b). Ces extrema ne doivent pas être confondus avec ceux dus à la fonction d'appareil.
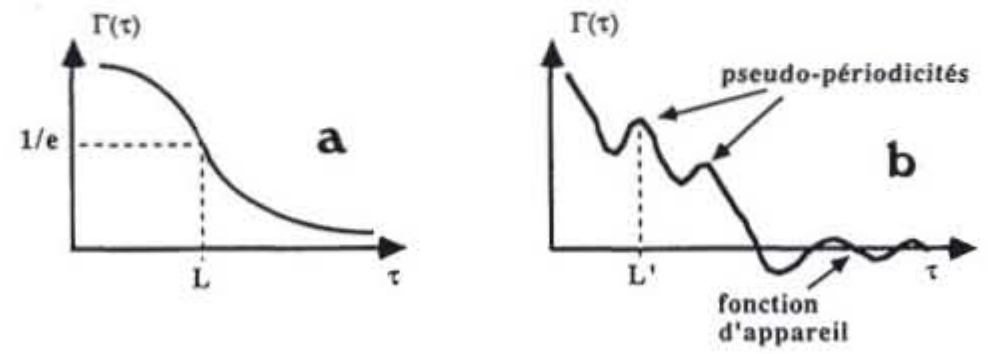

Fig.13: Fonctions d'autncorrélation pour une surface aléatoire (a) et pour une surface présentant des pseudo-périodicités(b).

Rappelons que la rugosité $\delta$ est donnée par la valeur à l'origine de la fonction d'autocorrélation:

$$
\delta^{2}=\Gamma(0)=\frac{1}{T} \int_{x} h^{2}(x) d x=\frac{1}{T} \int_{\sigma} \gamma(\sigma) d \sigma
$$




\section{2-Fonction de transfert}

Supposons que l'on puisse éclairer la surface avec une onde plane, c'est-à-dire une nappe de lumière infiniment étendue spatialement et donc non divergente. L'indicatrice de diffusion présente alors de fortes fluctuations angulaires (fig.14) qui sont imposées par le spectre de rugosité $\gamma(\vec{\sigma})=4 \pi^{2}|\hat{h}(\vec{\sigma})|^{2}$ du profil aléatoire $\mathrm{h}(\overrightarrow{\mathrm{r}})$. Cette indicatrice constitue le speckle "brut" (non perturbé par la mesure) intrinsèque au micropoli. D'un point de vue pratique, on éclaire avec un paquet d'ondes planes caractérisé par une répartition angulaire d'énergie $A^{2}(\ddot{\beta})$. On montre que le spectre $\hat{h}$ est convolué par la fonction $A$, ce qui conduit à un lissage du speckle. Cette courbe moyennée (fig.14) serait obtenue pour un détecteur situé à l'infini, c'est-à-dire en l'absence de perturbation due à l'angle solide de mesure.

Dans le cas d'un éclairement gaussien $\mathrm{e}^{-(\mathrm{r} / \mathrm{a})^{2}}$ au niveau de l'échantillon, $\mathrm{A}^{2}(\beta)$ est en $\mathrm{e}^{-(\beta a)^{2}}$ et le spectre $\hat{h}(\vec{\sigma})=\operatorname{TF}[h(\vec{r})]$ est moyenné sur une largeur fréquentielle $\Delta \sigma=\sqrt{2} /$ a ou angulaire $\Delta \theta=\lambda / \pi$ a $\sqrt{2}$ caractéristique de la divergence du faisceau incident. On voit donc qu'il n'est pas souhaitable de réduire la surface éclairée (de rayon a) au niveau de l'échantillon.

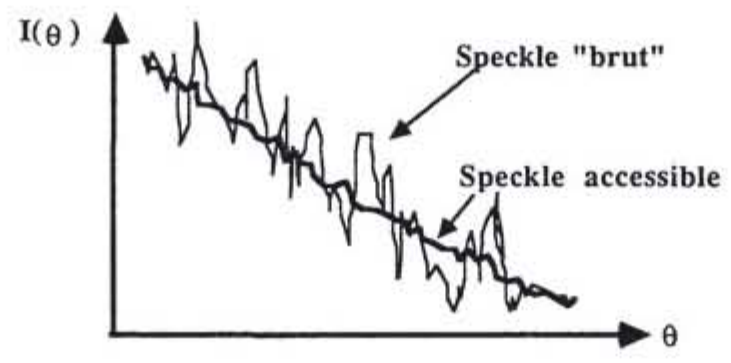

Fig.14: Speckle "brut" et convolué.

D'une façon plus générale, on voudrait accéder au spectre réel $\gamma(\vec{\sigma})$ de l'échantillon alors que la mesure donne accès à: $\gamma_{\mathrm{m}}(\vec{\sigma})=\left[4 \pi^{2}|\hat{\mathrm{h}} * \mathrm{~A}|^{2} * \Omega\right]$. Circ $\left(\frac{\sigma}{\mathrm{k}}\right)$ où $\Omega$ décrit l'effet de l'angle solide de mesure et Circ rappelle que la fréquence spatiale maximale mesurable vaut $k / 2 \pi=1 / \lambda$. De même, on accède à une fonction d'autocorrélation $\Gamma_{\mathrm{m}}$ donnée par: $\Gamma_{\mathrm{m}}(\vec{\tau})=[$ Autoc $(\mathrm{h} \hat{\mathrm{A}}) \hat{\Omega}] * \mathrm{TF}^{-1}\left(\operatorname{Circ}_{\mathrm{k}} \frac{\sigma}{)}\right.$. Cette fonction n'a de sens physique que sur une distance $\tau$ faible devant le support de $\hat{\Omega}$, d'où l'intérêt d'avoir un angle solide aussi faible que possible (il faut $\hat{\Omega} \approx 1$ ). En d'autres termes, une longueur d'autocorrélation $L$ mesurée sur un échantillon ne caractérise le micropoli que si $\mathrm{L} \ll\langle\Delta \Delta \theta$, où $\Delta \theta$ est relatif à l'angle solide de mesure. Quant au produit de convolution par la fonction $\operatorname{TF}^{-1}(\operatorname{Circ}(\sigma / k))$ de support proche de $\lambda$, il rappelle que la lumière ne voit pas les détails de dimensions latérales inférieures a la longueur d'onde. En figure 15, on donne un exemple d'autocorrélation relative à un micropoli; les oscillations sont dues à la fonction d'appareil du diffusomètre. On a tenu compte également de la fenêtre liée à l'angle minimum de mesure de la diffusion. 


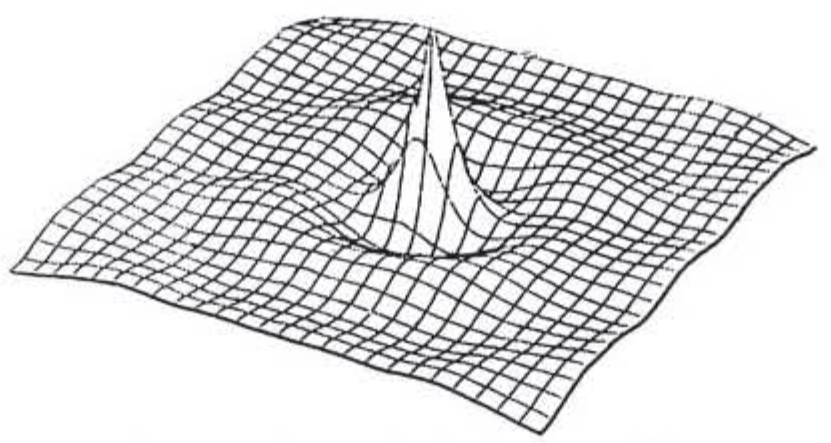

Fig.15: Exemple de mesure d'une fonction d'autocorrelation.

\section{3-Comparaison des méthodes optiques et mécaniques}

Pour les profilomètres, la fonction d'appareil dépend du rayon de courbure du stylet et de sa longueur de parcours sur l'échantillon. On peut utiliser le calcul numérique sur ordinateur pour comparer les "visions" optique et mécanique d'un état de surface. Cette comparaison est importante si l'on veut espérer tendre vers une normalisation de la caractérisation des micropolis (il faut ajuster les différentes fonctions d'appareils).

\section{VII-Anisotropie de surface}

Beaucoup de surfaces présentent des directions privilégiées qui sont responsables d'une dispersion de l'indicatrice de diffusion avec le plan polaire $\phi$. Ces surfaces sont dites anisotropes et créent une diffusion qui n'est pas de révolution autour de la normale à l'échantillon. Une façon de représenter cette anisotropie consiste à tracer des cartes de niveau en coordonnées polaires: tout écart par rapport à des cercles concentriques est caractéristique de l'anisotropie du micropoli (fig.16). Toutefois, il s'agit là d'une description qualitative et il est souhaitable d'aller plus loin dans la description de ce phénomène.
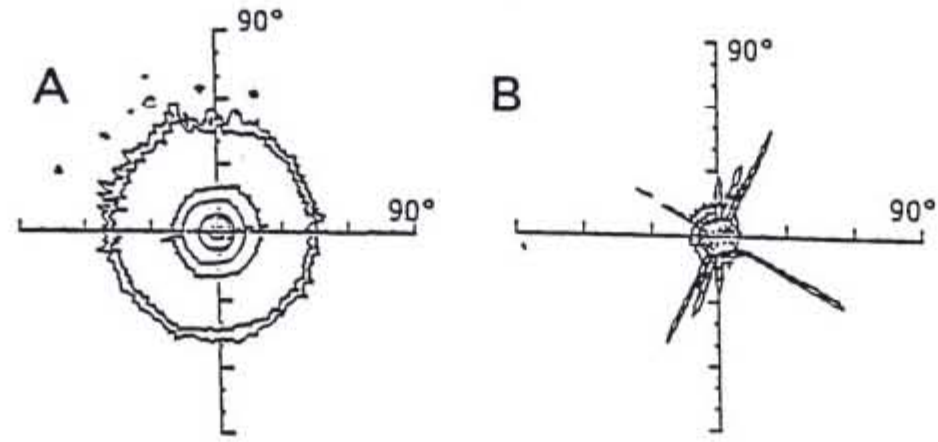

Fig.16: Courbes de niveau $(x=\theta \cos \phi$ et $y=\theta \sin \phi)$ de la diffusion par une surface isotrope (A) et anisotrope (B).

\section{1-Isotropie totale}

La surface $\mathrm{z}=\mathrm{h}(\overrightarrow{\mathrm{r}})$ est isotrope si le désordre est le même quelle que soit la direction considérée sur l'échantillon, c'est-à-dire si la fonction d'autocorrélation est radiale $: \Gamma(\vec{\tau})=\Gamma[|\vec{\tau}|]$. Pour étudier cette 
propriété, on introduit la fonction de corrélation angulaire $F(\alpha)=\int_{\vec{\tau}} \Gamma[\vec{\tau}] \Gamma\left[R_{\alpha}(\vec{\tau})\right] d \vec{\tau}$, où $R_{\alpha}$ désigne une rotation d'angle $\alpha$ dans le plan $\mathrm{z}=0$. Cette fonction exprime un état statistique de ressemblance entre la surface $\mathrm{h}(\overrightarrow{\mathrm{r}})$ et la même surface ayant subi une rotation dans son plan moyen. Le théorème de Parseval permet d'exprimer $F(\alpha)$ en fonction du spectre de rugosité de l'échantillon:

$$
\mathrm{F}(\alpha)=4 \pi^{2} \int_{\vec{\sigma}} \gamma(\vec{\sigma}) \gamma\left[R_{\alpha}(\vec{\sigma})\right] \mathrm{d} \vec{\sigma}=4 \pi^{2} \mathrm{k}^{2} \int_{\theta, \phi} \gamma(\theta, \phi) \gamma(\theta, \phi+\alpha) \sin \theta \cos \theta \mathrm{d} \theta \mathrm{d} \phi
$$

Puisque l'on sait mesurer le spectre $\gamma=\mathrm{I} / \mathrm{C}$, cette grandeur $\mathrm{F}(\alpha)$ est donc accessible à l'expérience. Remarquons que $F(\alpha)$ doit être symétrique par rapport à $\pi / 2$.

Pour une surface isotrope, la fonction normalisée $\mathrm{F}_{\mathrm{N}}(\alpha)=\mathrm{F}(\alpha) / \mathrm{F}(0)$ est constante et unitaire dans tout le domaine $0 \leq \alpha \leq 2 \pi$. Dans le cas contraire, ses variations sont caractéristiques du désordre en rotation. Les extrema locaux ou pseudo-périodicités angulaires sont liés à la présence, sur la surface rugueuse, de plusieurs directions privilégiées. On définit le degré d'isotropie d comme le minimum de $\mathrm{F}_{\mathrm{N}}(\alpha)$; c'est l'analogue de la longueur d'autocorrélation, d'un point de vue angulaire $(\mathrm{d}=1$ pour une surface isotrope). Toutefois, il s'agit là d'une grandeur intégrée (comme la rugosité) puiqu'elle tient compte simultanément de toutes les fréquences spatiales de défauts. Nous pouvons aller plus loin en définissant une anisotropie angulaire ou fréquentielle.

\section{2-Anisotropie angulaire}

L'intégrale de Fourier permet également de décomposer le profil h( $\overrightarrow{\mathrm{r}})$ de la façon suivante: $h(\vec{r})=\int_{\sigma} h_{\sigma}(\vec{r}) d \sigma$, où $h_{\sigma}$ exprime la contribution de tous les réseaux sinusoïdaux de même pas $c=2 \pi / \sigma$, mais d'orientations arbitraires. En d'autres termes, $\mathrm{h}_{\sigma}$ est la composante responsable de la diffusion dans un cône de demi-angle au sommet $\theta$ (avec $\sigma=k \sin \theta$ ). On sait calculer l'autocorrélation puis la corrélation angulaire de cette composante: $\mathrm{F}_{\sigma}(\alpha)=\mathrm{F}_{\theta}(\alpha)=4 \pi^{2} \sigma^{4} \int_{\phi} \gamma(\theta, \phi) \gamma(\theta, \phi+\alpha)$ d $\phi$ qui n'est autre que l'intégrale partielle sur l'angle polaire. En figure 17, on donne quelques exemples de mesure pour différentes directions $\theta$ de diffusion. Il est possible d'extraire les variations du degré d'isotropie avec la fréquence spatiale des défauts: $d(\theta)=\operatorname{Min}_{\alpha}\left(\mathrm{F}_{\mathrm{N}}(\theta, \alpha)\right.$ ). Ceci permet de caractériser dans le détail l'anisotropie de la rugosité, laquelle constitue une véritable signature de l'état de surface.

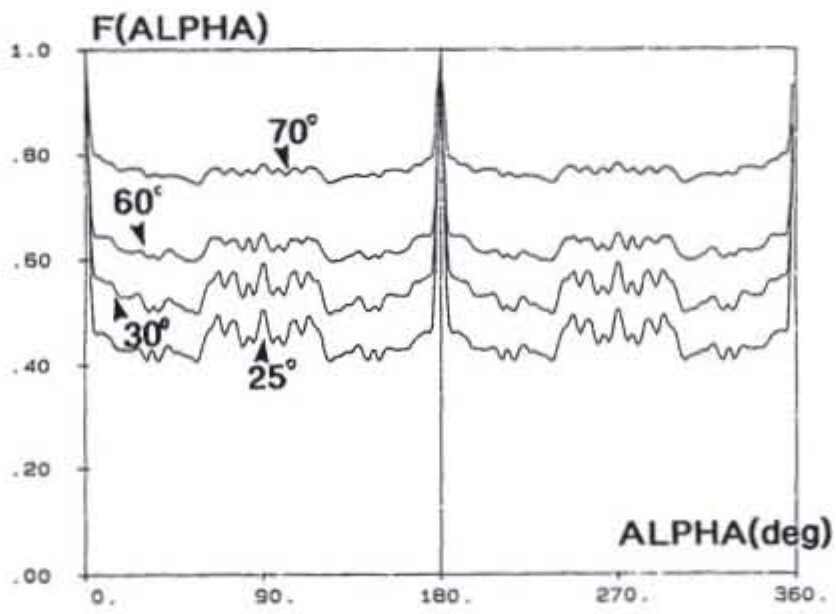

Fig.17: Correlations angulaires $F_{N}(\theta, \alpha)$ mesurées pour differents angles $\theta$ de diffusion. 


\section{VIII-Conclusion}

Ce cours était destiné à mettre en évidence les problèmes qui se posent lorsque l'on veut caractériser avec précision un état de surface. Cette caractérisation est essentielle si l'on veut pouvoir étudier les inhomogénéités de volume et rugosités résiduelles intrinsèques aux matériaux sous forme de couches minces déposées sur micropolis; par exemple, si les matériaux se contentent de reproduire (partiellement ou complètement) la rugosité du substrat, les variations fréquentielles du degré d'isotropie ne sont pas altérées par le revêtement multicouche: ceci permet de séparer nettement l'effet substrat des autres effets intrinsèques aux matériaux.

\section{Quelques références bibliographiques}

- J.M. Elson, J.P. Rahn, and J. Bennett, "Relationship of the total integrated scattering from multilayercoated optics to angle of incidence, polarization, correlation-length, and roughness crosscorrelation properties", Appl.Opt., 22, 3207 (1983).

-P. Roche, E. Pelletier, "Characterizations of optical surfaces by measurement of scattering distribution", Appl.Opt., 23, 3561-3566 (1984).

- P. Croce, L. Prod'homme, "Ecarts observés dans l'interprétation des indicatrices de diffusion optique par des théries vectorielles simples", J.Optics (Paris), 16, 143-151 (1985).

- C.Amra, G.Albrand, and P.Roche, "Theory and application of antiscattering single layers; antiscattering antireflection coatings", Appl.Opt., 25, 2695-2702 (1986).

- C.Amra, P. Bousquet, "Scattering from surfaces and multilayer coatings: recent advances for a better investigation of experiment", Proc.Soc.Photo-Opt.Instrum.Eng., "Surface Measurement and Characterization", 1009, 82-97 (1988).

- C.Grèzes-Besset, C.Amra, B.Cousin, G.Otrio, E.Pelletier, R.Richier, "Etude de la diaphonie d'un système de démultiplexage par filtres interférentiels. Conséquences de la diffusion de la lumière par les irrégularités des surfaces optiques", Ann.Télécommun., 43, 135-141 (1988).

- C.Amra, "Scattering characterization of materials in thin film form", "Laser induced damage in optical materials", 1438, 309-323 (1989).

- C. Amra, "Calculs et mesures de diffusion appliqués à l'étude de la rugosité dans les traitements optiques multicouches", J.Optics, 21, 83-98 (1990). 